See Article page 1269.

\section{Commentary: The benefits of going big: Cost savings and reduced mortality in a national study of proximal aortic repair}

\author{
Joseph S. Coselli, MD
}

The National Inpatient Sample (NIS) serves as a valuable resource to identify trends for hospitalized patients within the United States with regard to access, cost, outcomes, and other factors; when weighted, this readily available federal data reflect more than $97 \%$ of US patients, and a wide variety of medical procedures are tracked using administrative codes from the International Classification of Diseases (most recently the International Classification of Diseases Clinical Modification, 9th Revision, and International Classification of Diseases Clinical Modification, 10th Revision/ Procedure Classification System). ${ }^{1}$ As pertains to aortic surgery, contemporary analyses have largely focused on use and outcome trends for technique-driven and other studies of the descending thoracic, ${ }^{2-4}$ thoracoabdominal, ${ }^{5,6}$ and abdominal aorta ${ }^{7,8}$ as well as studies on aortic dissection. ${ }^{9-11}$ Although considerable effort has been made to better understand practice trends in repair of the distal aorta (ie, the descending thoracic, thoracoabdominal, and abdominal aorta), there are a lack of NIS-based studies that exclusively examine the proximal aorta, namely the aortic root, ascending aorta, and aortic arch.

Hirji and colleagues from Harvard Medical School ${ }^{12}$ aim to improve our understanding of national trends as it pertains specifically to the proximal aorta. Based on an NIS sample of 53,657 adult patients undergoing open repair of the aortic root, ascending aorta or aortic arch between

\footnotetext{
From the Division of Cardiothoracic Surgery, Michael E. DeBakey Department of Surgery, Baylor College of Medicine; Department of Cardiovascular Surgery, Texas Heart Institute; and CHI St Luke's Health-Baylor St Luke's Medical Center, Houston, Tex.

Disclosures: Dr Coselli's work is partly supported by the Cullen Foundation. Dr Coselli consults for, receives royalties and a departmental educational grant from, and participates in clinical trials for Terumo Aortic; consults and participates in clinical trials for Medtronic, Inc, and W.L. Gore \& Associates; and serves as a coinvestigator for CytoSorbents.

Received for publication March 19, 2020; accepted for publication March 20, 2020; available ahead of print April 10, 2020.

Address for reprints: Joseph S. Coselli, MD, One Baylor Plaza, BCM 390, Houston, TX 77030 (E-mail: jcoselli@bcm.edu).

J Thorac Cardiovasc Surg 2022;163:1281-2

$0022-5223 / \$ 36.00$

Copyright $(2020$ by The American Association for Thoracic Surgery

https://doi.org/10.1016/j.jtcvs.2020.03.067
}

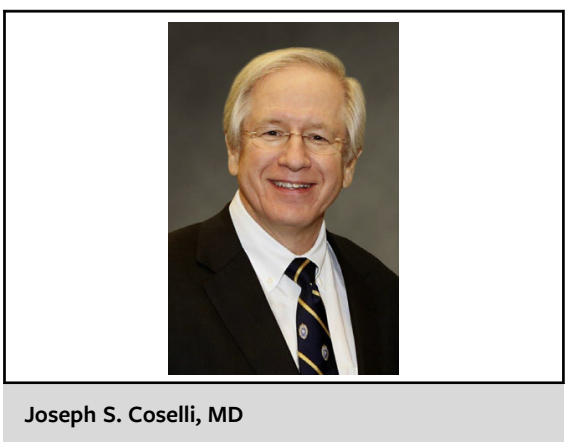

CENTRAL MESSAGE

For patients undergoing proximal aortic repair, national data indicate a trend toward improved outcomes and lower costs_changes driven by large hospitals.

2002 and 2014, the authors uncovered several notable findings. When analyzing operative mortality over time, there was more than a $50 \%$ reduction in death from 2002 to 2014 , as rates dropped from $13.9 \%$ to $6.7 \%$, respectively. During the same time period, there was a dramatic increase of nearly $40 \%$ in the sheer number of procedures performed. Of these procedures, the vast majority $(>75 \%)$ were performed at large hospitals as compared with hospitals designated as small $(6 \%)$ or medium $(16 \%)$. When examining the role that hospital size played in patient outcomes, they found that larger hospitals tended to care for sicker and older patients but were able to achieve similar outcomes at a substantially lower cost $(>15 \mathrm{~K})$ and with a shorter hospital stay than did smaller hospitals. Although part of this savings reflects a greater number of patients being discharged from large hospitals to rehabilitation facilities (3\% more), even after factoring in these related costs, the savings are likely pronounced and may reflect the systemic advantages of larger hospitals as pertains to discharge pathways. Regarding predictors of operative mortality, it comes as no surprise that the presence of rupture is the greatest predictor of death (3.5 times enhanced risk); hospital size was not a factor. In this study, improvement appeared primarily driven by nondissection repairs conducted in large hospitals.

Analysis of NIS data is notoriously difficult and may be best used to capture trends. As a systemic evaluation by Khera and colleagues ${ }^{13}$ determined, although the 
availability of large data sets is undoubtedly attractive to researchers, it is difficult for most studies to be performed in accordance to conduct standards. Within the study by Hirji and coauthors, a propensity-matched study of 9242 pairs found few notable differences by hospital size; this may reflect the difficulty in truly matching patients when the primary type of aortic repair (ie, aortic root vs ascending aortic vs aortic arch repair) remains unknown. In addition, there are other curious findings in this study, including a relatively low rate of elective procedures, a pronounced rate of aortic dissection (as contrasted against an expected rate of aortic rupture), and a substantial difference in patient age across hospital size. However, any limitations of this study are muted by the pronounced drop in operative mortality within a relatively short time period; in an era of disruptive technology such as what endovascular aortic repair unleashed, it is good comfort to know that benchmark mortality rates for complex aortic repair are trending substantially lower. Go big or go home; large hospital systems show the way.

\section{References}

1. Overview of the National (Nationwide) Inpatient Sample (NIS). Available at: https://www.hcup-us.ahrq.gov/nisoverview.jsp. Accessed March 17, 2020.

2. Delafontaine JL, Hu B, Tan TW, Tang GL, Starnes BW, Virk C, et al. Outcome com parison of TEVAR with and without left subclavian artery revascularization from analysis of nationwide inpatient sample database. Ann Vasc Surg. 2019;58:174-9.

3. Hughes K, Guerrier J, Obirieze A, Ngwang D, Rose D, Tran D, et al. Open versus endovascular repair of thoracic aortic aneurysms: a nationwide inpatient sample study. Vasc Endovascular Surg. 2014;48:383-7.
4. Gopaldas RR, Huh J, Dao TK, LeMaire SA, Chu D, Bakaeen FG, et al. Superior nationwide outcomes of endovascular versus open repair for isolated descending thoracic aortic aneurysm in 11,669 patients. J Thorac Cardiovasc Surg. 2010; 140:1001-10.

5. Arnaoutakis DJ, Propper BW, Black JH III, Schneider EB, Lum YW, Freischlag JA, et al. Racial and ethnic disparities in the treatment of unruptured thoracoabdominal aortic aneurysms in the United States. J Surg Res. 2013;184: 651-7.

6. Liao JM, Bakaeen FG, Cornwell LD, Simpson K, Lemaire SA, Coselli JS, et al. Nationwide trends and regional/hospital variations in open versus endovascular repair of thoracoabdominal aortic aneurysms. J Thorac Cardiovasc Surg. 2012;144:612-6.

7. Gupta PK, Ramanan B, Grossman L, Gupta H, Fang X, MacTaggart JN, et al. Outcomes of aortic surgery for abdominal aortic graft infections. Vasc Endovascular Surg. 2016;50:256-60.

8. Dua A, Furlough CL, Ray H, Sharma S, Upchurch GR, Desai SS. The effect of hospital factors on mortality rates after abdominal aortic aneurysm repair. $J$ Vasc Surg. 2014;60:1446-51.

9. Wang GJ, Jackson BM, Foley PJ, Damrauer SM, Goodney PP, Kelz RR, et al. National trends in admissions, repair, and mortality for thoracic aortic aneurysm and type B dissection in the national inpatient sample. J Vasc Surg. 2018;67:1649-58.

10. Zimmerman KP, Oderich G, Pochettino A, Hanson KT, Habermann EB, Bower TC, et al. Improving mortality trends for hospitalization of aortic dissection in the national inpatient sample. J Vasc Surg. 2016;64: 606-15.e1.

11. Chikwe J, Cavallaro P, Itagaki S, Seigerman M, DiLuozzo G, Adams D, et al. National outcomes in acute aortic dissection: influence of surgeon and institutional volume on operative mortality. Ann Thorac Surg. 2013; 95:1563-9.

12. Hirji S, Shah R, Aranki S, McGurk S, Singh S, Mallidi H, et al. The impact of hospital size on national trends and outcomes in isolated open proximal surgery. J Thorac Cardiovasc Surg. 2022;163:1269-78.e9.

13. Khera R, Angraal S, Couch T, Welsh JW, Nallamothu BK, Girotra S, et al. Adherence to methodological standards in research using the national inpatient sample. JAMA. 2017;318:2011-8. 